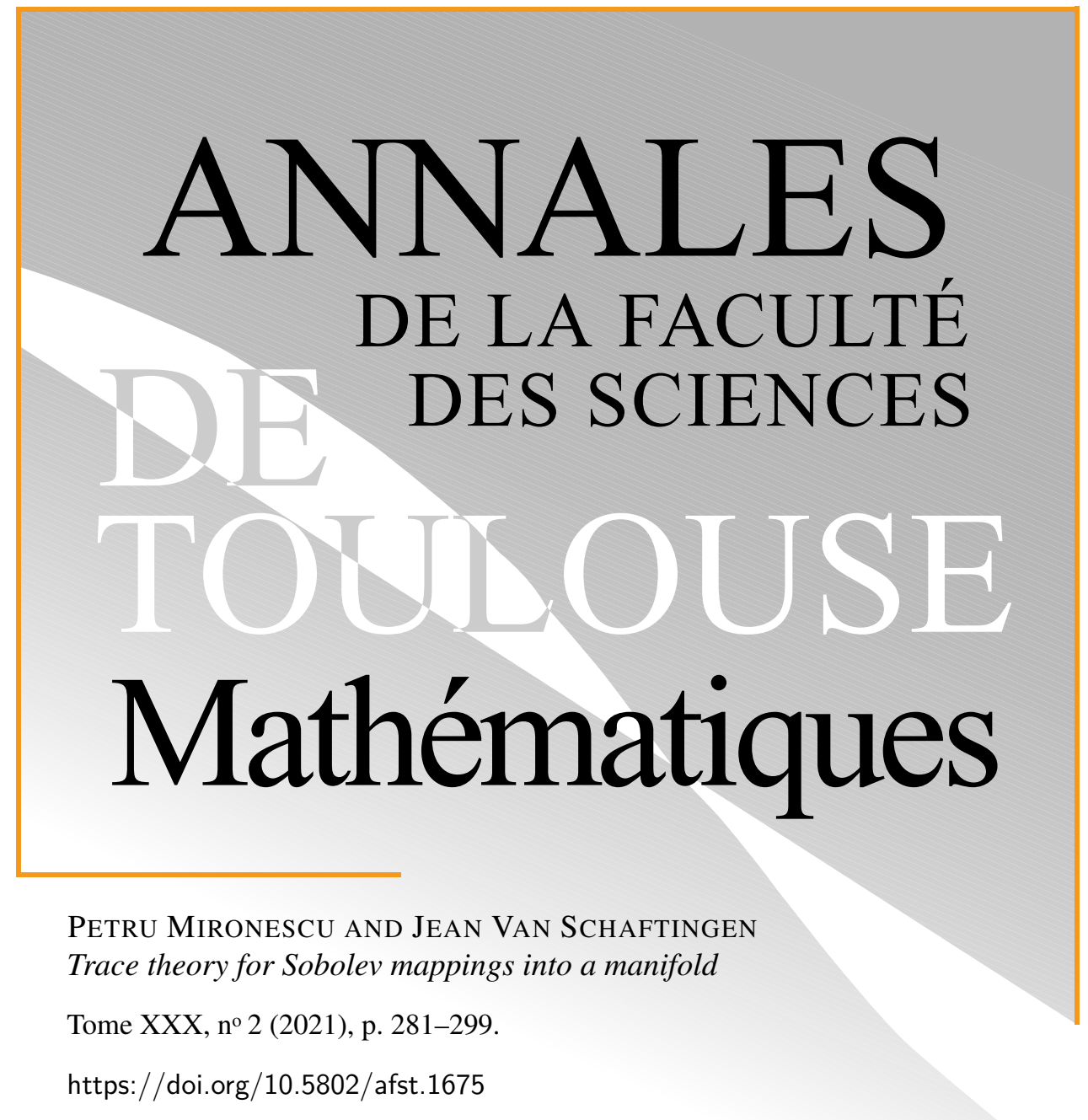

Petru Mironescu and Jean Van Schaftingen

Trace theory for Sobolev mappings into a manifold

Tome XXX, nº 2 (2021), p. 281-299.

https://doi.org/10.5802/afst.1675

(c) Université Paul Sabatier, Toulouse, 2021.

L'accès aux articles de la revue «Annales de la faculté des sciences de Toulouse Mathématiques» (http://afst.centre-mersenne.org/) implique l'accord avec les conditions générales d'utilisation (http://afst.centre-mersenne.org/legal/). Les articles sont publiés sous la license CC-BY 4.0.

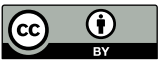

Publication membre du centre

Mersenne pour l'édition scientifique ouverte 


\title{
Trace theory for Sobolev mappings into a manifold
}

\author{
Petru Mironescu ${ }^{(1)}$ And Jean Van Schaftingen ${ }^{(2)}$
}

\begin{abstract}
We review the current state of the art concerning the characterization of traces of the spaces $W^{1, p}\left(\mathbb{B}^{m-1} \times(0,1), \mathcal{N}\right)$ of Sobolev mappings with values into a compact manifold $\mathcal{N}$. In particular, we exhibit a new analytical obstruction to the extension, which occurs when $p<m$ is an integer and the homotopy group $\pi_{p}(\mathcal{N})$ is non trivial. On the positive side, we prove the surjectivity of the trace operator when the fundamental group $\pi_{1}(\mathcal{N})$ is finite and $\pi_{2}(\mathcal{N}) \simeq \cdots \simeq \pi_{\lfloor p-1\rfloor}(\mathcal{N}) \simeq\{0\}$. We present several open problems connected to the extension problem.

RÉSuMÉ. - Nous examinons l'état de l'art de la caractérisation des traces des espaces $W^{1, p}\left(\mathbb{B}^{m-1} \times(0,1), \mathcal{N}\right)$ d'applications Sobolev à valeurs valeurs dans une variété compacte $\mathcal{N}$. En particulier, nous mettons en évidence une nouvelle obstruction analytique à l'extension, qui se produit lorsque $p<m$ est un entier et que le groupe d'homotopie $\pi_{p}(\mathcal{N})$ n'est pas trivial. Du côté positif, nous démontrons la surjectivité de l'opérateur de trace lorsque le groupe fondamental $\pi_{1}(\mathcal{N})$ est fini et que $\pi_{2}(\mathcal{N}) \simeq \cdots \simeq \pi_{\lfloor p-1\rfloor}(\mathcal{N}) \simeq\{0\}$. Nous présentons plusieurs problèmes ouverts liés au problème d'extension.
\end{abstract}

Keywords: Trace spaces, fractional Sobolev spaces, homotopy groups, lifting of Sobolev mappings.

2010 Mathematics Subject Classification: 46T10, 46E35, 58D15.

(1) Université Lyon 1, CNRS UMR 5208 Institut Camille Jordan, 43 Boulevard du 11 Novembre 1918, 69622 Villeurbanne Cedex, France, and, Simion Stoilow Institute of Mathematics of the Romanian Academy, Calea Griviţei 21, 010702 Bucureşti, România — mironescu@math.univ-lyon1.fr

(2) Université catholique de Louvain, Institut de Recherche en Mathématique et Physique, Chemin du Cyclotron 2 bte L7.01.01, 1348 Louvain-la-Neuve, Belgium jean.vanschaftingen@uclouvain.be

This work has been initiated during a long term visit of P. Mironescu at the Simion Stoilow Institute of Mathematics of the Romanian Academy; he thanks the Institute and the Centre Francophone en Mathématiques in Bucharest for their support on that occasion.

J. Van Schaftingen was supported by the Mandat d'Impulsion Scientifique F.4523.17, "Topological singularities of Sobolev maps" of the Fonds de la Recherche Scientifique-FNRS. 


\section{Introduction}

The classical trace theory characterizes the boundary values of functions in the linear Sobolev spaces $W^{1, p}\left(\mathbb{R}^{m-1} \times(0,1), \mathbb{R}\right)$, with $m \geqslant 2$ and $1 \leqslant$ $p<\infty$. These spaces are defined as

$$
W^{1, p}\left(\mathbb{R}^{m-1} \times(0,1), \mathbb{R}\right) \triangleq\left\{U: \mathbb{R}^{m-1} \times(0,1) \rightarrow \mathbb{R} ; U \in L^{p} \text { and } D U \in L^{p}\right\},
$$

where $D U$ is weak derivative of $U$.

The characterization of the traces involves the fractional SobolevSlobodeckiu space $W^{1-1 / p, p}\left(\mathbb{R}^{\ell}, \mathbb{R}\right)$. Recall that, when $0<s<1$, the fractional spaces $W^{s, p}\left(\mathbb{R}^{\ell}, \mathbb{R}\right)$ are defined as

$$
W^{s, p}\left(\mathbb{R}^{\ell}, \mathbb{R}\right) \triangleq\left\{u: \mathbb{R}^{\ell} \rightarrow \mathbb{R} ; u \in L^{p} \text { and } \mathcal{E}^{s, p}(u)<\infty\right\},
$$

where the fractional Gagliardo energy $\mathcal{E}^{s, p}(u)$ of a measurable function $u$ : $\mathbb{R}^{\ell} \rightarrow \mathbb{R}$ is given by

$$
\mathcal{E}^{s, p}(u) \triangleq \iint_{\mathbb{R}^{\ell} \times \mathbb{R}^{\ell}} \frac{|u(y)-u(x)|^{p}}{|y-x|^{\ell+s p}} \mathrm{~d} y \mathrm{~d} x .
$$

The fractional spaces $W^{s, p}\left(\mathbb{R}^{\ell}, \mathbb{R}\right)$ can also be characterized as interpolated spaces between $L^{p}\left(\mathbb{R}^{\ell}, \mathbb{R}\right)$ and $W^{1, p}\left(\mathbb{R}^{\ell}, \mathbb{R}\right)$ [20, Théorème VI.2.1] (see also [1, Theorem 7.39]).

The central result in classical trace theory, due to E. Gagliardo [16] (see also [15, §10.17-10.18 and Proposition 17.1], [21, §10.1.1]), asserts that, when $p>1$, there exists a unique linear continuous surjective trace operator $\operatorname{tr}_{\mathbb{R}^{m-1} \times\{0\}}: W^{1, p}\left(\mathbb{R}^{m-1} \times(0,1), \mathbb{R}\right) \rightarrow W^{1-1 / p, p}\left(\mathbb{R}^{m-1}, \mathbb{R}\right)$, extending the (pointwise) trace on $\mathbb{R}^{m-1} \times\{0\}$ of smooth maps $U \in C^{\infty}\left(\mathbb{R}^{m-1} \times[0,1), \mathbb{R}\right) \cap$ $W^{1, p}\left(\mathbb{R}^{m-1} \times(0,1), \mathbb{R}\right)$. Moreover, the operator $\operatorname{tr}_{\mathbb{R}^{m-1} \times\{0\}}$ has a linear continuous right inverse. The harmonic extension (convolution with the Poisson kernel), the heat semigroup (convolution with the heat kernel) or, more generally, the convolution with appropriate families of mollifiers are explicit examples of such right inverses. For example, if $u \in W^{1-1 / p, p}\left(\mathbb{R}^{m-1}, \mathbb{R}\right)$, then its harmonic extension $U$ to $\mathbb{R}^{m-1} \times(0, \infty)$, restricted to $\mathbb{R}^{m-1} \times(0,1)$, is an extension of $u$ in the sense that it belongs to $W^{1, p}\left(\mathbb{R}^{m-1} \times(0,1), \mathbb{R}\right)$ and has trace $u$ on $\mathbb{R}^{m-1} \times\{0\}$.

When $p=1$, the trace operator is a linear continuous surjection on $L^{1}\left(\mathbb{R}^{m-1}\right)[16]$ that has no linear continuous right inverse (J. Peetre [27]).

Trace theory has local versions, in which the whole Euclidean space $\mathbb{R}^{m-1}$ is replaced by a Lipschitz domain. For simplicity, we focus on the case of the unit ball $\mathbb{B}^{m-1}$. With $1 \leqslant p<\infty$ and $0<s<1$, the adapted Sobolev spaces 
and fractional energies are

$$
\begin{gathered}
W^{1, p}\left(\mathbb{B}^{m-1} \times(0,1), \mathbb{R}\right) \triangleq\left\{U: \mathbb{B}^{m-1} \times(0,1) \rightarrow \mathbb{R} ; U \in L_{\mathrm{loc}}^{1} \text { and } D U \in L^{p}\right\}, \\
W^{s, p}\left(\mathbb{B}^{\ell}, \mathbb{R}\right) \triangleq\left\{u: \mathbb{R}^{\ell} \rightarrow \mathbb{R} ; u \text { is measurable and } \mathcal{E}^{s, p}(u)<\infty\right\},
\end{gathered}
$$

and

$$
\mathcal{E}^{s, p}(u) \triangleq \iint_{\mathbb{B}^{\ell} \times \mathbb{B}^{\ell}} \frac{|u(y)-u(x)|^{p}}{|y-x|^{\ell+s p}} \mathrm{~d} y \mathrm{~d} x .
$$

In this framework, when $p>1$, the trace operator

$$
\operatorname{tr}_{\mathbb{B}^{m-1} \times\{0\}}: W^{1, p}\left(\mathbb{B}^{m-1} \times(0,1), \mathbb{R}\right) \rightarrow W^{1-1 / p, p}\left(\mathbb{B}^{m-1}, \mathbb{R}\right)
$$

is a linear continuous surjection that has a linear continuous right inverse. Again, explicit extensions of maps in $W^{1-1 / p, p}\left(\mathbb{B}^{m-1}, \mathbb{R}\right)$ can be obtained via convolutions with appropriate mollifiers.

The previous considerations extend readily to the case where the target space $\mathbb{R}$ is replaced by a finite-dimensional Euclidean space $\mathbb{R}^{\nu}$, where $\nu \in \mathbb{N}_{*}$.

When $\mathcal{N} \subset \mathbb{R}^{\nu}$ is an embedded compact Riemannian submanifold ${ }^{(1)}$, we consider the corresponding Sobolev spaces of mappings into the manifold $\mathcal{N}$, defined, for $1 \leqslant p<\infty$ and $0<s<1$, as

$$
\begin{aligned}
& W^{1, p}\left(\mathbb{B}^{m-1} \times(0,1), \mathcal{N}\right) \\
& \triangleq\left\{U \in W^{1, p}\left(\mathbb{B}^{m-1} \times(0,1), \mathbb{R}^{\nu}\right) ; U \in \mathcal{N} \text { almost everywhere in } \mathbb{B}^{m-1} \times(0,1)\right\}
\end{aligned}
$$

and

$$
W^{s, p}\left(\mathbb{B}^{\ell}, \mathcal{N}\right) \triangleq\left\{u \in W^{s, p}\left(\mathbb{B}^{\ell}, \mathbb{R}^{\nu}\right) ; u \in \mathcal{N} \text { almost everywhere in } \mathbb{B}^{\ell}\right\} .
$$

The classical linear theory readily implies that

$$
\operatorname{tr}_{\mathbb{B}^{m-1} \times\{0\}}\left(W^{1, p}\left(\mathbb{B}^{m-1} \times(0,1), \mathcal{N}\right)\right) \subseteq W^{1-1 / p, p}\left(\mathbb{B}^{m-1}, \mathcal{N}\right)
$$

(with the convention $W^{0,1}\left(\mathbb{B}^{m-1}, \mathcal{N}\right)=L^{1}\left(\mathbb{B}^{m-1}, \mathcal{N}\right)$ ).

The basic question of the trace and extension theory for Sobolev mappings with values into manifolds is to determine whether equality holds in the inclusion (1.1); the linear trace theory merely provides an extension taking its values into the ambient Euclidean space $\mathbb{R}^{\nu}$ and the problem is to determine whether every map $u \in W^{1-1 / p, p}\left(\mathbb{B}^{m-1}, \mathcal{N}\right)$ has a $W^{1, p}\left(\mathbb{B}^{m-1} \times(0,1), \mathbb{R}^{\nu}\right)$ extension with values into $\mathcal{N}$. If this holds, then $W^{1-1 / p, p}\left(\mathbb{B}^{m-1}, \mathcal{N}\right)$ has the extension property.

(1) By Nash's embedding theorem [26], such an embedding exists for any abstract Riemannian manifold. 
Let us start by noting a harmless condition in order to study the extension property: the manifold $\mathcal{N}$ will be connected. Indeed, if $U \in W_{\text {loc }}^{1,1}\left(\mathbb{B}^{m-1} \times\right.$ $(0,1), \mathcal{N})$, then the essential range of the map $U$ is connected [11, Theorem 7.5], and thus the mapping $U$ takes values into a connected component of $\mathcal{N}$; therefore, so does its trace. When $p<2$, there exists a map $u \in W^{1-1 / p, p}\left(\mathbb{B}^{m-1}, \mathcal{N}\right)$ that takes constant values on smooth subsets of $\mathbb{B}^{m-1}$, and therefore we have to assume that the manifold $\mathcal{N}$ is necessarily connected. On the other hand when $p \geqslant 2$, the essential range of any map $u \in W^{1-1 / p, p}\left(\mathbb{B}^{m-1}, \mathcal{N}\right)$ is connected and there is thus no loss of generality to work with a connected target manifold $\mathcal{N}$. We assume henceforth that the manifold $\mathcal{N}$ is connected.

In the case of subcritical dimensions $m \leqslant p$, the answer to the trace and extension problem is positive.

THEOREM 1.1. - If $m \leqslant p$, then $W^{1-1 / p, p}\left(\mathbb{B}^{m-1}, \mathcal{N}\right)$ has the extension property.

Theorem 1.1 is due to F. Bethuel and F. Demengel [7, Theorems 1 \& 2]. Its proof relies on the fact that, when $p \geqslant m$, an extension by convolution of a map $u \in W^{1-1 / p, p}\left(\mathbb{B}^{m-1}, \mathcal{N}\right)$ takes, in a neighborhood of $\mathbb{B}^{m-1} \times\{0\}$, its values in a small tubular neighborhood of $\mathcal{N}$. This important observation has roots in the seminal work of R. Schoen and K. Uhlenbeck [30, §3], [31, §4] on $H^{1}$ maps with values into manifolds; see also H. Brezis and L. Nirenberg [14] for far-reaching consequences of properties of this type in connection with the degree theory for vanishing mean oscillation (VMO) maps with values into manifolds.

In higher dimensions $m>p$, the answer to the trace problem is also positive provided the integrability exponent $p$ is small.

THEOREM 1.2. - If $1 \leqslant p<2$, then $W^{1-1 / p, p}\left(\mathbb{B}^{m-1}, \mathcal{N}\right)$ has the extension property.

Theorem 1.2 is due to is due to R. Hardt and F.H. Lin [17, Theorem 6.2$] \cdot{ }^{(2)}$

(2) Strictly speaking, the case $p=1$, which is an exceptional case for trace theory, is not specifically considered in [17]. However, Theorem 1.2 with $p=1$ and Theorem 1.9(2) are proved exactly as the corresponding results for $1<p<2$ [7, Proof of Theorem 7 (i)]. The initial ingredient is the existence, for each measurable map $u: \mathbb{B}^{m-1} \rightarrow \mathcal{N}$, of some extension $U \in W^{1,1}\left(\mathbb{B}^{m-1} \times(0,1), \mathbb{R}^{\nu}\right)$ such that

$$
\|\nabla U\|_{L^{1}} \leqslant C\left\|u-f_{\mathbb{B}^{m-1}} u\right\|_{L^{1}} .
$$


In particular, when $m=2$, the whole range of integrability exponents $1 \leqslant p<\infty$ is covered by the combination of Theorem 1.1 and Theorem 1.2. A hint to the absence of any topological condition beyond connectedness of the manifold $\mathcal{N}$ is the fact that, when $0<s p<1$, the space $W^{s, p}\left(\mathbb{B}^{m-1}, \mathbb{R}^{\nu}\right)$ contains characteristic functions of smooth sets and hence topological obstructions cannot arise in these spaces. (A similar phenomenon arises for the lifting problem when $0<s p<1[5,8]$.)

However, when $2 \leqslant p<m$, one encounters some obstructions in the extension problem. A first example is provided by the topological obstruction.

THEOREM 1.3. - If $2 \leqslant p<m$ and if $\pi_{\lfloor p-1\rfloor}(\mathcal{N}) \not\left\{\{0\}^{(3)}\right.$, then $W^{1-1 / p, p}\left(\mathbb{B}^{m-1}, \mathcal{N}\right)$ does not have the extension property.

Theorem 1.3 is due to R. Hardt and F.H. Lin $[17, \S 6.3]$ and F. Demengel and F. Bethuel [7, Theorem 4]. An equivalent formulation of the above topological obstruction is the following: there exists a map $f \in C^{1}(\mathbb{S}\lfloor p-1\rfloor, \mathcal{N})$ that cannot be extended continuously to the ball $\mathbb{B}^{\lfloor p\rfloor}$. Given such an $f$, an explicit example of a map $u \in W^{1-1 / p, p}\left(\mathbb{B}^{m-1}, \mathcal{N}\right)$ with no extension $U \in W^{1, p}\left(\mathbb{B}^{m-1} \times(0,1), \mathcal{N}\right)$ is given by

$$
\begin{aligned}
u\left(x^{\prime}, x^{\prime \prime}\right) \triangleq & f\left(x^{\prime} /\left|x^{\prime}\right|\right), \\
& \forall\left(x^{\prime}, x^{\prime \prime}\right) \in \mathbb{R}^{\lfloor p\rfloor} \times \mathbb{R}^{m-1-\lfloor p\rfloor} \text { such that }\left(x^{\prime}, x^{\prime \prime}\right) \in \mathbb{B}^{m-1} .
\end{aligned}
$$

By Theorem 1.3 above, in the range $2 \leqslant p<m$, a necessary condition for the extension property to hold is $\pi_{\lfloor p-1\rfloor}(\mathcal{N}) \simeq\{0\}$. When $2 \leqslant p<3 \leqslant m$, this condition becomes $\pi_{1}(\mathcal{N}) \simeq\{0\}$, i.e., $\mathcal{N}$ has to be simply connected. This condition turns out to be also sufficient.

THEOREM 1.4. - If $2 \leqslant p<3 \leqslant m$ and if $\pi_{1}(\mathcal{N}) \simeq\{0\}$, then $W^{1-1 / p, p}\left(\mathbb{B}^{m-1}, \mathcal{N}\right)$ has the extension property.

Theorem 1.4 is due to R. Hardt and F.H. Lin [17, Theorem 6.2].

Besides the topological obstruction, the extension problem encounters some analytical obstructions.

Theorem 1.5. - Assume $2 \leqslant p<m$. If

(a) either $\pi_{\ell}(\mathcal{N})$ is infinite for some $\ell \in\{1, \ldots,\lfloor p-1\rfloor\}$

(b) or $p \in \mathbb{N}$ and $\pi_{p-1}(\mathcal{N}) \nsucceq\{0\}$,

then there exists some smooth map $u \in W^{1-1 / p, p}\left(\mathbb{B}^{m-1}, \mathcal{N}\right)$ that has no extension $U \in W^{1, p}\left(\mathbb{B}^{m-1} \times(0,1), \mathcal{N}\right)$. In particular, $W^{1-1 / p, p}\left(\mathbb{B}^{m-1}, \mathcal{N}\right)$ does not have the extension property.

(3) Here and in what follows, $\lfloor t\rfloor \in \mathbb{Z}$ denotes the integer part of the real number $t \in \mathbb{R}$. 
Theorem 1.5(a) is due to F. Demengel and F. Bethuel when $\ell=1[7$, Theorem 4] and to F. Bethuel for a general $\ell$ [4]. ${ }^{(4)}$ Theorem 1.5(b) is one of the contributions of the present work (see Section 2 below).

The map $u$ given in Theorem 1.5 is not smooth up to the boundary. However, $u$ is the strong limit of maps smooth up to the boundary. Note the difference in nature with the counterexample (1.2) in Theorem 1.3; there, $u$ has strong interior singularities in the set $\mathbb{B}^{m-1} \cap\left(\{0\} \times \mathbb{R}^{m-1-\lfloor p\rfloor}\right)$.

When $p$ is an integer, Theorem 1.5(b) implies that the assumption that $\pi_{p-1}(\mathcal{N})$ is trivial plays, in the extension problem, a role even for the strong limits of smooth maps (and is not only required just to have the strong density of smooth maps $[10,13])$.

On the positive side, we have the following result.

THEOREM 1.6. - If $3 \leqslant p<m$, if $\pi_{1}(\mathcal{N})$ is finite and if $\pi_{2}(\mathcal{N}) \simeq \cdots \simeq$ $\pi_{\lfloor p-1\rfloor} \simeq\{0\}$, then $W^{1-1 / p, p}\left(\mathbb{B}^{m-1}, \mathcal{N}\right)$ has the extension property.

Theorem 1.6 is due to R. Hardt and F.H. Lin [17, Theorem 6.2] when $\pi_{1}(\mathcal{N})$ is trivial. In full generality, it is proved in the present work (see Section 3 below). The proof strongly relies on an idea of F. Bethuel [4, Theorem 1.5(iii)] and uses a very recent result on the lifting over compact covering spaces [24].

Combining Theorems 1.2, 1.3, 1.4, 1.5 and 1.6, we obtain the following.

Corollary 1.7. - Assume $m \geqslant p$.

(1) If $1 \leqslant p<2$, then $W^{1-1 / p, p}\left(\mathbb{B}^{m-1}, \mathcal{N}\right)$ has the extension property.

(2) If $2 \leqslant p<3$, then $W^{1-1 / p, p}\left(\mathbb{B}^{m-1}, \mathcal{N}\right)$ has the extension property if and only if $\pi_{1}(\mathcal{N}) \simeq\{0\}$.

(3) If $3 \leqslant p<4$, then $W^{1-1 / p, p}\left(\mathbb{B}^{m-1}, \mathcal{N}\right)$ has the extension property if and only if $\pi_{1}(\mathcal{N})$ is finite and $\pi_{2}(\mathcal{N}) \simeq\{0\}$.

What happens when $4 \leqslant p<m$ (assuming the necessary conditions for the extension property imposed by Theorem 1.3 and Theorem 1.5) is terra incognita.

Open problem 1.8. - Assume $4 \leqslant p<m, \pi_{1}(\mathcal{N}), \ldots, \pi_{\lfloor p-2\rfloor}(\mathcal{N})$ finite and $\pi_{\lfloor p-1\rfloor}(\mathcal{N})$ trivial. Does $W^{1-1 / p, p}\left(\mathbb{B}^{m-1}, \mathcal{N}\right)$ have the extension property?

(4) The triviality of the groups $\pi_{1}(\mathcal{N}), \ldots, \pi_{\ell-1}(\mathcal{N})$ and the non-triviality of $\pi_{\ell}(\mathcal{N})$ (which are the only explicit assumptions in [4]) do not imply that $\pi_{\ell}(\mathcal{N})$ is infinite; see Proposition 4.1. However, the latter property is used in the construction of maps with arbitrary large topological energy [4, Lemma 2.2]. 
F. Bethuel and F. Demengel have conjectured the answer to Open Problem 1.8 to be positive [7, Conjecture 2]. Let us note that there exist manifolds satisfying the assumptions of Open Problem 1.8 (see Proposition 4.1).

We next turn to the quantitative form of the extension problem, more specifically the existence of $U$ whose energy is controlled in terms of the one of $u$. Given $u \in W^{1-1 / p, p}\left(\mathbb{B}^{m-1}, \mathcal{N}\right)$, a natural extension energy is

$$
\mathcal{E}_{\text {ext }}^{1, p}(u) \triangleq \inf \left\{\int_{\mathbb{B}^{m-1} \times(0,1)}|\mathrm{D} U|^{p} ; U \text { is an extension of } u\right\} .
$$

The next result shows that, under the topological assumptions in Theorem 1.2, Theorem 1.4 or Theorem 1.6, the extension energy is controlled linearly.

THEOREM 1.9 .

(1) If

(a) either $1<p<2$,

(b) or $2 \leqslant p<3$ and $\pi_{1}(\mathcal{N}) \simeq\{0\}$,

(c) or $3 \leqslant p<\infty, \pi_{1}(\mathcal{N})$ is finite and $\pi_{2}(\mathcal{N}) \simeq \cdots \simeq \pi_{\lfloor p-1\rfloor}(\mathcal{N}) \simeq\{0\}$, then there exists a constant $C=C(p, m, \mathcal{N})$ such that, for every mapping $u \in W^{1-1 / p, p}\left(\mathbb{B}^{m-1}, \mathcal{N}\right)$,

$$
\mathcal{E}_{\text {ext }}^{1, p}(u) \leqslant C \mathcal{E}^{1-1 / p, p}(u) .
$$

(2) If $p=1$, then

$$
\mathcal{E}_{\mathrm{ext}}^{1,1}(u) \leqslant C\left\|u-f_{\mathbb{B}^{m-1}} u\right\|_{L^{1}} .
$$

Theorem 1.9 is a direct consequence of the estimates resulting from the proofs of Theorems 1.2, 1.4 and 1.6. Note that we do not require $p<m$. In the range $1<p<m$, Theorem 1.9 follows without any calculation from the existence results in the above theorems and an abstract nonlinear uniform boundedness principle due to A. Monteil and J. Van Schaftingen [25, Theorem 1.1].

On the other hand, the topology of $\mathcal{M}$ can create obstructions to linear estimates on the extension, even for smooth maps.

Theorem 1.10. - Assume that $\ell \in\{1, \ldots, m-1\}$. Let $b \in \mathcal{N}$. If

(a) either $\ell<p-1$ and $\pi_{\ell}(\mathcal{N})$ is infinite,

(b) or $\ell=p-1$ and $\pi_{\ell}(\mathcal{N})$ is nontrivial, 
then there exists a sequence $\left(u_{j}\right)_{j \in \mathbb{N}}$ in $C_{b}^{\infty}\left(\overline{\mathbb{B}}^{m-1}, \mathcal{N}\right)^{(5)}$ such that

$$
\liminf _{j \rightarrow \infty} \mathcal{E}^{1-1 / p, p}\left(u_{j}\right)>0 \quad \text { and } \quad \lim _{j \rightarrow \infty} \frac{\mathcal{E}_{\text {ext }}^{1, p}\left(u_{j}\right)}{\mathcal{E}^{1-1 / p, p}\left(u_{j}\right)}=\infty .
$$

Case (a) in Theorem 1.10 is due to Bethuel [4]; (b) one of the contributions of the present work (see Section 2 below). Theorem 1.9 and Theorem 1.10 leave an open question about quantitative estimates:

OPEN PROBLEM 1.11. - If $p \geqslant 4$, and $\pi_{1}(\mathcal{N}), \ldots, \pi_{\lfloor p-2\rfloor}(\mathcal{N})$ are finite and if $\pi_{\lfloor p-1\rfloor}(\mathcal{N})$ is trivial, does there exist a constant $C$ such that for every $u \in W^{1-1 / p, p}\left(\mathbb{B}^{m-1}, \mathcal{N}\right)$, one has

$$
\mathcal{E}_{\text {ext }}^{1, p}(u) \leqslant C \mathcal{E}^{1-1 / p, p}(u) ?
$$

In the cases where the trace operator is not surjective, a natural question is to describe the elements in the trace space, in a similar fashion to what has been done in many cases for the strong approximation by smooth maps of Sobolev mappings [2, 6, 28].

Open PROBLEM 1.12. - Characterize the trace space

$$
\operatorname{tr}_{\mathbb{B}^{m-1} \times\{0\}}\left(W^{1, p}\left(\mathbb{B}^{m-1} \times(0,1), \mathcal{N}\right)\right) .
$$

When either $1 \leqslant p<2$ or $p \geqslant m$, then by Theorems 1.1 and 1.2 the trace space is the fractional Sobolev space $W^{1-1 / p, p}\left(\mathbb{B}^{m-1}, \mathcal{N}\right)$. When $2 \leqslant p<3$, a map $u$ is in the trace space if and only $u$ has a $W^{1-1 / p, p}$ lifting in the universal covering of $\mathcal{N}$. ${ }^{(6)}$ (This assertion can be established by adapting the proof of Theorem 3 in [4]; see also Section 3.) However, currently there is no tractable characterization of the mappings having this property.

A partial result in this direction has been obtained by $\mathrm{B}$. White $[33$, Theorem 4.1], who characterized maps in $\operatorname{tr}_{\mathbb{B}^{m-1} \times\{0\}}\left(W^{1, p}\left(\mathbb{B}^{m-1} \times(0,1), \mathcal{N}\right)\right)$ that are in addition Lipschitz-continuous.

When $p \in \mathbb{N}$, the trace spaces can be characterized by a topological condition on generic skeletons and the boundedness of families of GinzburgLandau energies remaining bounded when the order parameter goes to 0 $[9,19]$; it would be desirable to have a more intrinsic criterion, probably relying on the behaviour of the map on $\lfloor p-2\rfloor$-dimensional skeletons. In view of the quantitative obstructions to the extension problem [4], the

(5) Here and in what follows, the subscript $b$ denotes classes of maps with trace $b$ on the boundary.

(6) More precisely, let $\widetilde{\mathcal{N}}$ be the universal covering of $\mathcal{N}$ and $\pi: \widetilde{\mathcal{N}} \rightarrow \mathcal{N}$ the corresponding covering map. Then $u \in \operatorname{tr}_{\mathbb{B}^{m-1} \times\{0\}}\left(W^{1, p}\left(\mathbb{B}^{m-1} \times(0,1), \mathcal{N}\right)\right)$ if and only if there exists some $\varphi \in W^{1-1 / p, p}\left(\mathbb{B}^{m-1}, \widetilde{\mathcal{N}}\right)$ such that $u=\pi \circ \varphi$. 
condition should be quantitative, in contrast with the more qualitative criteria for the strong approximation by smooth maps.

Up to this point, we have considered the problem of traces on $\mathbb{B}^{m-1} \times\{0\}$ of maps on $\mathbb{B}^{m-1} \times(0,1)$. More generally, one can consider a manifold $\mathcal{M}$ with a boundary $\partial \mathcal{M}$ and traces on $\partial \mathcal{M}$.

Open PROBLEM 1.13. - When do we have

$$
\operatorname{tr}_{\partial \mathcal{M}}\left(W^{1, p}(\mathcal{M}, \mathcal{N})\right)=W^{1-1 / p, p}(\partial \mathcal{M}, \mathcal{N}) ?
$$

R. Hardt and F.H. Lin have proved that this is the case when $\pi_{1}(\mathcal{N}) \simeq$ $\cdots \simeq \pi_{\lfloor p-1\rfloor}(\mathcal{N}) \simeq\{0\}$ [17, Theorem 6.2]. On the other hand, if $\operatorname{tr}_{\partial \mathcal{M}}\left(W^{1, p}(\mathcal{M}, \mathcal{N})\right)=W^{1-1 / p, p}(\partial \mathcal{M}, \mathcal{N})$, then $\mathcal{M}$ and $\mathcal{N}$ have to satisfy the following topological property: For some arbitrary ${ }^{(7)}$ triangulation $\mathcal{T}$ of the manifold $\mathcal{M}$, every $\mathcal{N}$-valued continuous map on the $\lfloor p-1\rfloor$-skeleton of $\mathcal{T} \cap \partial \mathcal{M}$ admits a continuous $\mathcal{N}$-valued extension to the $\lfloor p\rfloor$-skeleton of $\mathcal{T}$ (T. Isobe [19]; see also [7, Theorem 5]).

The linear trace theory extends to weighted spaces [32] (see also [23]): if one sets

$$
\begin{aligned}
& W_{\gamma}^{1, p}\left(\mathbb{B}^{m-1} \times(0,1)\right) \\
& \quad=\left\{U \in W_{\text {loc }}^{1,1}\left(\mathbb{B}^{m-1} \times(0,1)\right) ; \iint_{\mathbb{B}^{m-1} \times(0,1)}|\mathrm{D} U(x, t)|^{p} t^{\gamma} \mathrm{d} t \mathrm{~d} x<\infty\right\},
\end{aligned}
$$

then, for $0<s<1$ and $1 \leqslant p<\infty$, we have

$$
\operatorname{tr}_{\mathbb{B}^{m-1} \times\{0\}} W_{(1-s) p-1}^{1, p}\left(\mathbb{B}^{m-1} \times(0,1)\right)=W^{s, p}\left(\mathbb{B}^{m-1}\right) .
$$

Open problem 1.14. - Assume $0<s<1$ and $1 \leqslant p<\infty$. Characterize the manifolds $\mathcal{N}$ for which one has

$$
\operatorname{tr}_{\mathbb{B}^{m-1} \times\{0\}} W_{(1-s) p-1}^{1, p}\left(\mathbb{B}^{m-1} \times(0,1), \mathcal{N}\right)=W^{s, p}\left(\mathbb{B}^{m-1}, \mathcal{N}\right) .
$$

Finally, if one considers higher-order Sobolev spaces, the derivatives also have traces. It is known for instance that, for $1 \leqslant p<\infty$, we have

$$
\begin{aligned}
\left\{\left(\operatorname{tr}_{\mathbb{B}^{m-1} \times\{0\}} U, \operatorname{tr}_{\mathbb{B}^{m-1} \times\{0\}} \partial_{m} U\right) ; U\right. & \left.\in W^{2, p}\left(\mathbb{B}^{m-1} \times(0,1)\right)\right\} \\
& =W^{2-1 / p, p}\left(\mathbb{B}^{m}\right) \times W^{1-1 / p, p}\left(\mathbb{B}^{m}\right) .
\end{aligned}
$$

Open PRoBlem 1.15. - Characterize the manifolds $\mathcal{N}$ such that

$$
\begin{aligned}
&\left\{\left(\operatorname{tr}_{\mathbb{B}^{m-1}} \times\{0\}\right.\right.\left.\left.U, \operatorname{tr}_{\mathbb{B}^{m-1} \times\{0\}} \partial_{m} U\right) ; U \in W^{2, p}\left(\mathbb{B}^{m-1} \times(0,1), \mathcal{N}\right)\right\} \\
&=W^{2-1 / p, p}\left(\mathbb{B}^{m}, \mathcal{N}\right) \times W^{1-1 / p, p}\left(\mathbb{B}^{m}, T \mathcal{N}\right) .
\end{aligned}
$$

(7) A homotopy equivalence argument shows that the condition does not depend on the triangulation. 


\section{Obstructions and non-estimates}

We first prove Theorem 1.10 about the obstruction to linear bounds on the extension energy $\mathcal{E}_{\text {ext }}^{1, p}$.

Towards the proof of Theorem 1.10: A fundamental lower bound when $p>\ell+1$. - We explain the main idea in [4]. For the convenience of the reader, we first consider maps defined on a cylinder, then we adapt the proof to the case of maps defined on half-balls.

Given mappings $u, v \in C\left(\overline{\mathbb{B}}^{\ell}, \mathcal{N}\right)$ we consider the following relative homotopy equivalence: $v \sim u$ if and only if there exists some $H \in C\left(\overline{\mathbb{B}}^{\ell} \times[0,1], \mathcal{N}\right)$ such that $H(\cdot, 0)=u, H(\cdot, 1)=v$ and $H(x, t)=u(x), \forall x \in \mathbb{S}^{\ell-1}, \forall t \in[0,1]$.

We consider some $U \in W^{1, p}\left(\mathbb{B}^{\ell} \times(0,1), \mathcal{N}\right)$ such that $\operatorname{tr}_{\mathbb{S}^{\ell-1} \times(0,1)} U=b .^{(8)}$ Identifying $U$ with its continuous representative ${ }^{(9)}$, we may assume that $U \in C\left(\overline{\mathbb{B}}^{\ell} \times[0,1], \mathcal{N}\right)$. Set $u(x) \triangleq U(x, 0), \forall x \in \overline{\mathbb{B}}^{\ell}$. By standard trace theory, we have $U(\cdot, t)=b$ on $\mathbb{S}^{\ell-1}, \forall t \in[0,1]$, and

$$
\operatorname{tr}_{\mathbb{B}^{\ell} \times\{0\}} U=U \uparrow_{\mathbb{B}^{\ell} \times\{0\}} \sim u .
$$

It follows that

$$
U(\cdot, \tau) \sim u, \forall \tau \in[0,1]
$$

(through the homotopy $[0,1] \ni t \mapsto U(\cdot,(1-t) \tau)$ ).

We next deduce a lower bound for the energy of $U$ as follows. By the Sobolev-Morrey embedding, we have

$$
W^{1, p}\left(\mathbb{B}^{\ell}, \mathcal{N}\right) \subset W^{1-1 / p, p}\left(\mathbb{B}^{\ell}, \mathcal{N}\right) \subset C^{0,1-(\ell+1) / p}\left(\overline{\mathbb{B}}^{\ell}, \mathcal{N}\right) .
$$

On the other hand, by standard trace theory the above $u$ satisfies $u \in$ $W^{1-1 / p, p}\left(\mathbb{B}^{\ell}, \mathcal{N}\right)$. By $(2.2)$, the quantity

$$
\mathcal{E}_{\text {top }}^{1, p}(u) \triangleq \inf \left\{\mathcal{E}^{1, p}(v) ; v \in W^{1, p}\left(\mathbb{B}^{\ell}, \mathcal{N}\right) \text { and } v \sim u\right\}
$$

is meaningful. Combining (2.1) with the fact that $U(\cdot, \tau) \in W^{1, p}\left(\mathbb{B}^{\ell}, \mathcal{N}\right)$ for almost every $\tau \in(0,1)$, we find that

$$
\mathcal{E}^{1, p}(U(\cdot, \tau)) \geqslant \mathcal{E}_{\text {top }}^{1, p}(u) \text { for almost every } \tau \in(0,1) .
$$

We next present an analogue of the above on a half-ball. Set $\mathbb{B}_{+}^{\ell+1} \triangleq$ $\mathbb{B}^{\ell+1} \cap \mathbb{R}_{+}^{\ell+1}$. We define similarly $\mathbb{S}_{+}^{\ell}$, and set

$$
S_{+}(0, r)=\left\{x \in \mathbb{R}^{\ell+1} ;|x|=r \text { and } x_{\ell+1}>0\right\} .
$$

(8) Recall that $b \in \mathcal{N}$ is a fixed point.

(9) This is possible, by the Morrey embedding, since $p>\ell+1$. 
Let $S$ be the South pole of $\mathbb{S}^{\ell}$ and let $\Psi$ denote the stereographic projection with vertex $S$ of $\mathbb{S}^{\ell} \backslash\{S\}$ on $\mathbb{R}^{\ell} \times\{0\} \simeq \mathbb{R}^{\ell}$. Thus $\Psi$ maps $\mathbb{S}_{+}^{\ell}$ onto $\mathbb{B}^{\ell}$ and leaves invariant $\mathbb{S}^{\ell-1} \times\{0\} \simeq \mathbb{S}^{\ell-1}$. Moreover, $\Psi$ is a bi-Lipschitz homeomorphism from $\overline{\mathbb{S}}_{+}^{\ell}$ to $\overline{\mathbb{B}}^{\ell}$.

Let $U \in W^{1, p}\left(\mathbb{B}_{+}^{\ell+1}, \mathcal{N}\right)$. As above, we may assume that $U \in C\left(\overline{\mathbb{B}_{+}^{\ell+1}}, \mathcal{N}\right)$. We set $u(x) \triangleq U(x, 0), \forall x \in \overline{\mathbb{B}}^{\ell}$ and, for $0<r \leqslant 1, U^{r}(x) \triangleq U\left(r \Psi^{-1}(x)\right)$, $\forall x \in \overline{\mathbb{B}}^{\ell}$, so that $U^{r} \in C\left(\overline{\mathbb{B}}^{\ell}, \mathcal{N}\right)$. Assume that the map $u$ has the property that

We claim that

$$
u(x)=b \text { for } \rho \leqslant|x| \leqslant 1 .
$$

$$
U^{r} \sim u, \forall \rho \leqslant r \leqslant 1 .
$$

Indeed, on the one hand we have $U^{1} \sim u=U(\cdot, 0)$ through the homotopy

$$
H(x, t)=U\left(t \Psi^{-1}(x)+(1-t)(x, 0)\right), \forall x \in \overline{\mathbb{B}}^{\ell}, \forall t \in[0,1] .
$$

On the other hand, we have, for $\rho \leqslant r, r^{\prime} \leqslant 1, U^{r} \sim U^{r^{\prime}}$ through $H(\cdot, t)=$ $U^{t r+(1-t) r^{\prime}}, \forall t \in[0,1] .{ }^{(10)}$ Combining this with the definition of $U^{r}$, we obtain the following analogue of (2.3):

$$
\int_{S_{+}(0, r)}\left|\nabla_{T} U\right|^{p} \geqslant C_{1} r^{\ell-p} \mathcal{E}_{\text {top }}^{1, p}(u), \text { for almost every } \rho<r<1 ;
$$

here, $C_{1}>0$ is an absolute constant, and $\nabla_{T}$ stands for the tangential gradient on the half-sphere $S_{+}(0, r)$.

Integrating the estimate (2.6), we find that

$$
\int_{\mathbb{B}_{+}^{\ell+1}}|\mathrm{D} U|^{p} \geqslant C_{1} \int_{\rho}^{1} r^{\ell-p} \mathrm{~d} r \mathcal{E}_{\mathrm{top}}^{1, p}(u) .
$$

Taking into account the fact that $\mathbb{B}_{+}^{\ell+1} \subset \mathbb{B}^{\ell} \times(0,1)$, (2.7) leads to the following fundamental lower bound.

$$
\begin{aligned}
& \mathcal{E}_{\text {ext }}^{1, p}(u) \geqslant C_{1} \mathcal{E}_{\text {top }}^{1, p}(u) \int_{\rho}^{1} r^{\ell-p} \mathrm{~d} r \\
& \forall u \in W^{1-1 / p, p}\left(\mathbb{B}^{\ell}, \mathcal{N}\right) \text { satisfying (2.4). }
\end{aligned}
$$

Proof of Theorem 1.10 when $p>\ell+1$ and $m=\ell+1$. - Since the homotopy group $\pi_{\ell}(\mathcal{N})$ is infinite, there exists a sequence $\left(v_{j}\right)_{j \in \mathbb{N}}$ in $C^{\infty}\left(\overline{\mathbb{B}}^{\ell}, \mathcal{N}\right)$ such that each $v_{j}$ is constant on $\mathbb{S}^{\ell-1}$ and $v_{j}$ is not homotopic with $v_{k}$ if $j \neq k$. Since $\mathcal{N}$ is connected and $v_{j}$ is constant on $\mathbb{S}^{\ell-1}$, we may assume

(10) Here, we use (2.4). 
with no loss of generality that $v_{j}=b$ on $\mathbb{S}^{\ell-1}, \forall j$. Consider now some map $w_{j} \in C_{b}^{\infty}\left(\overline{\mathbb{B}}^{\ell}, \mathcal{N}\right)$ such that $w_{j} \sim v_{j}$ and

$$
\mathcal{E}^{1, p}\left(w_{j}\right) \leqslant 2 \mathcal{E}_{\text {top }}^{1, p}\left(v_{j}\right)=2 \mathcal{E}_{\text {top }}^{1, p}\left(w_{j}\right) .
$$

This is clearly possible, from the definition of $\mathcal{E}_{\text {top }}^{1, p}$ and the density of $C_{b}^{\infty}\left(\overline{\mathbb{B}}^{\ell}, \mathcal{N}\right)$ in $W_{b}^{1, p}\left(\overline{\mathbb{B}}^{\ell}, \mathcal{N}\right) .{ }^{(11)}$ We claim that

$$
\lim _{j \rightarrow \infty} \mathcal{E}^{1-1 / p, p}\left(w_{j}\right)=\infty .
$$

Indeed, argue by contradiction and assume that (2.10) does not hold. Using the Morrey type embedding $W^{1-1 / p, p}\left(\mathbb{B}^{\ell}\right) \subset C^{0,1-(\ell+1) / p}\left(\overline{\mathbb{B}}^{\ell}\right)$, we find that, up to a subsequence, the sequence $\left(w_{j}\right)_{j \in \mathbb{N}}$ converges uniformly on $\overline{\mathbb{B}}^{\ell}$, and thus for large $j$ and $k$ we have $w_{j} \sim w_{k}$, which is impossible.

We next modify $w_{j}$ by setting

$$
u_{j}(x) \triangleq \begin{cases}w_{j}(2 x) & \text { if }|x| \leqslant 1 / 2, \\ b & \text { if }|x|>1 / 2,\end{cases}
$$

and note that $u_{j} \sim w_{j} \sim v_{j}$. From the above, $u_{j}$ satisfies (2.4) with $\rho=1 / 2$ and, in addition,

$$
\mathcal{E}^{1, p}\left(u_{j}\right) \leqslant C_{2} \mathcal{E}_{\text {top }}^{1, p}\left(u_{j}\right)
$$

and

$$
\lim _{j \rightarrow \infty} \mathcal{E}^{1-1 / p, p}\left(u_{j}\right)=\infty .
$$

To summarize, if $\pi_{\ell}(\mathcal{N})$ is infinite and $p>\ell+1$, then there exists a sequence $\left(u_{j}\right)_{j \in \mathbb{N}}$ in $C_{b}^{\infty}\left(\overline{\mathbb{B}}^{\ell}, \mathcal{N}\right)$ satisfying $(2.11),(2.12)$ and $(2.4)$ with $\rho=1 / 2$.

We next invoke the following fractional Gagliardo-Nirenberg type inequality

$$
\begin{aligned}
\mathcal{E}^{1-1 / p, 1}(u) \leqslant C_{3}\left[\mathcal{E}^{1, p}(u)\right]^{1-1 / p}\|u\|_{L^{\infty}}^{1 / p} \leqslant C_{4}\left[\mathcal{E}^{1, p}(u)\right]^{1-1 / p} & \\
& \forall u \in W^{1, p}\left(\mathbb{B}^{\ell}, \mathcal{N}\right)
\end{aligned}
$$

(see e.g. [29, Lemma 2.1], [12, Corollary 3.2], [22]). Combining (2.8), (2.11) and (2.13), we obtain the following superlinear lower bound

$$
\mathcal{E}_{\text {ext }}^{1, p}\left(u_{j}\right) \geqslant C_{5}\left[\mathcal{E}^{1-1 / p, p}\left(u_{j}\right)\right]^{p /(p-1)}, \forall j .
$$

We obtain the conclusion of the theorem from (2.14) and (2.12). (Strictly speaking, the mapping $u_{j}$ is only Lipschitz-continuous. However, using a

(11) Recall that $p>\ell$ and thus $W^{1, p}\left(\mathbb{B}^{\ell}, \mathcal{N}\right) \subset C\left(\overline{\mathbb{B}}^{\ell}, \mathcal{N}\right)$. Using this, the density of $C_{b}^{\infty}\left(\overline{\mathbb{B}}^{\ell}, \mathcal{N}\right)$ in $W^{1, p}\left(\mathbb{B}^{\ell}, \mathcal{N}\right)$ is straightforward; see e.g. [3, Introduction], and also the proof of Theorem 1.10 when $p=\ell+1$. 
standard approximation procedure, we obtain a sequence $\left(u_{j}\right) \subset C_{b}^{\infty}\left(\overline{\mathbb{B}}^{\ell}, \mathcal{N}\right)$ such that (2.14) and (2.12) hold.)

Proof of Theorem 1.10 when $p>\ell+1$ and $m>\ell+1$. - The main idea is to proceed to a dimensional reduction. To illustrate this, consider the maps $f_{j}(y, z) \triangleq u_{j}(y), \forall(y, z) \in \mathbb{B}^{\ell} \times(0,1)^{m-\ell-1}$ (with $u_{j}$ as above). Via a Fubini type argument, it is easy to see that

$$
\mathcal{E}_{\text {ext }}^{1, p}\left(f_{j}\right) \geqslant \mathcal{E}_{\text {ext }}^{1, p}\left(u_{j}\right) .
$$

On the other hand, by a direct calculation we have

$$
C_{6} \mathcal{E}^{1-1 / p, p}\left(u_{j}\right) \leqslant \mathcal{E}^{1-1 / p, p}\left(f_{j}\right) \leqslant C_{7} \mathcal{E}^{1-1 / p, p}\left(u_{j}\right) .
$$

Combining (2.15)-(2.16) with the properties of $u_{j}$, we find that $f_{j}$ satisfies (1.4).

However, however $f_{j}$ does not equal $b$ on $\mathbb{S}^{\ell-1}$. In order to obtain a map with this additional property, we replace, in the above construction, $(0,1)^{m-\ell-1}$ with a convenient sphere. The main ingredient is the existence of some $\Phi \in C^{\infty}\left(\overline{\mathbb{B}}^{\ell} \times \mathbb{S}^{m-\ell-1}, \mathbb{B}^{m-1}\right)$ such that $\Phi$ is a diffeomorphism into its image $V$. Taking this for granted, we argue as follows. Let $u_{j}$ be as above, and set $g_{j}(y, z) \triangleq u_{j}(y), \forall y \in \overline{\mathbb{B}}^{\ell}, \forall z \in \mathbb{S}^{m-\ell-1}$, and

$$
h_{j}(x) \triangleq \begin{cases}g_{j}\left(\Phi^{-1}(x)\right) & \text { if } x \in V, \\ b & \text { if } x \in \overline{\mathbb{B}}^{m-1} \backslash V .\end{cases}
$$

Then $h_{j} \in C_{b}^{\infty}\left(\overline{\mathbb{B}}^{m-1}, \mathcal{N}\right)$. By adapting the arguments leading to $(2.15)$ and (2.16), we find that $\left(h_{j}\right)_{j \in \mathbb{N}}$ has the required properties.

It remains to prove the existence of $\Phi$. Consider, for $z \in \mathbb{S}^{m-\ell-1}$, the following vectors in $\mathbb{R}^{m-1}$ :

$$
X_{1} \triangleq(z, 0), X_{2} \triangleq e_{m-\ell+1}, \ldots, X_{\ell} \triangleq e_{m-1} .
$$

Then, for sufficiently small $\varepsilon>0$, the mapping

$$
\overline{\mathbb{B}}^{\ell} \times \mathbb{S}^{m-\ell-1} \ni(y, z) \mapsto \Phi(y, z) \triangleq \frac{1}{2}(z, 0)+\varepsilon \sum_{k=1}^{\ell} y_{k} X_{k} \in \mathbb{R}^{m-1}
$$

has the required properties.

Proof of Theorem 1.10 when $p=\ell+1$. - As explained above, it suffices to consider the case $m=\ell+1$.

(12) Here, $\left(e_{1}, \ldots, e_{m-1}\right)$ is the canonical base of $\mathbb{R}^{m-1}$. 
If we examine the proof of (2.8), we see that the following lower bound is valid for any $p$. If

$$
u \in W^{1-1 / p, p}\left(\mathbb{B}^{\ell}, \mathcal{N}\right) \cap C_{b}\left(\overline{\mathbb{B}}^{\ell}, \mathcal{N}\right)
$$

satisfies (2.4), then

$$
\begin{aligned}
& \int_{\mathbb{B}_{+}^{\ell+1}}|\mathrm{D} U|^{p} \geqslant C_{1} \int_{\rho}^{1} r^{\ell-p} \mathrm{~d} r \mathcal{E}_{\text {top }}^{1, p}(u), \\
& \quad \forall U \in W^{1, p}\left(\mathbb{B}_{+}^{\ell+1}, \mathcal{N}\right) \cap C\left(\overline{\mathbb{B}_{+}^{\ell+1}}, \mathcal{N}\right) \text { such that } \operatorname{tr}_{\mathbb{B}^{\ell} \times\{0\}} U=u .
\end{aligned}
$$

The key observation is that, when $p=\ell+1$ and $u$ is, in addition, Lipschitzcontinuous, (2.18) holds even if $U$ is not supposed continuous, i.e.,

$$
\begin{aligned}
\int_{\mathbb{B}_{+}^{\ell+1}}|\mathrm{D} U|^{p} \geqslant & C_{1} \mathcal{E}_{\text {top }}^{1, p}(u) \int_{\rho}^{1} r^{-1} \mathrm{~d} r \\
& \forall U \in W^{1, \ell+1}\left(\mathbb{B}_{+}^{\ell+1}, \mathcal{N}\right) \text { such that } \operatorname{tr}_{\mathbb{B}^{\ell} \times\{0\}} U=u .
\end{aligned}
$$

This is obtained by proving that (under these assumptions on $p$ and $u$ ) for any map $U \in W^{1, p}\left(\mathbb{B}_{+}^{\ell+1}, \mathcal{N}\right)$ such that $\operatorname{tr}_{\mathbb{B}^{\ell} \times\{0\}} U \sim u$ there exists a sequence $\left(U_{j}\right)_{j \in \mathbb{N}}$ of mappings in $C^{\infty}\left(\overline{\mathbb{B}_{+}^{\ell+1}}, \mathcal{N}\right)$ such that $U_{j} \rightarrow U$ strongly in $W^{1, p}, U_{j}(\cdot, 0) \sim u$ for large $u$ and $U_{j}(x, 0)=b$ if $\rho \leqslant|x| \leqslant 1$.

Here is a sketch of proof of this fact, well-known to experts and reminiscent from the theory of vanishing mean oscillation (VMO) maps with values into manifolds (see H. Brezis and L. Nirenberg [14]). First, we extend $U$ to $\mathbb{B}^{\ell+1} \backslash \mathbb{B}_{+}^{\ell+1}$ by setting $U(x, t)=u(x)$ if $t \leqslant 0$. Next, we extend $U$ by reflection across $\mathbb{S}^{\ell}$. We may thus assume that $U \in W^{1, \ell+1}(B(0,3 / 2), \mathcal{N})$. We next consider $V_{j}(x, t) \triangleq U((1+1 / j) x, t-1 / j)$. For large $j, V_{j}$ is defined in $B(0,4 / 3)$ and satisfies $V_{j}(x, t)=u((1+1 / j) x)$ if $|x| \leqslant 5 / 4$ and $|t| \leqslant 1 / j$. In addition, we have $V_{j}(x, t)=b$ if $\rho /(1+1 / j) \leqslant|x| \leqslant 1+1 / j$ and $|t| \leqslant 1 / j$. Consider now a standard mollifier $\zeta \in C_{c}^{\infty}\left(\overline{\mathbb{B}}^{\ell+1}, \mathbb{R}\right)$ and let $\Pi$ denote the nearest point projection on $\mathcal{N}$. Then $\Pi\left(V_{j} * \zeta_{\varepsilon}\right) \rightarrow V_{j}$ in $W^{1, \ell+1}\left(\mathbb{B}_{+}^{\ell+1}, \mathcal{N}\right)$ as $\varepsilon \rightarrow 0$ [14]. ${ }^{(13)}$ We easily find that, for a suitable sequence $\varepsilon_{j} \rightarrow 0, U_{j} \triangleq \Pi\left(V_{j} * \zeta_{\varepsilon_{j}}\right)$ has all the required properties.

We complete the case $p=\ell+1$ and $m=\ell+1$ as follows. Since $\pi_{\ell}(\mathcal{N}) \not$ $\{0\}$, there exists a map $v \in C_{b}^{\infty}\left(\overline{\mathbb{B}}^{\ell}, \mathcal{N}\right)$ such that $v=b$ near $\mathbb{S}^{\ell-1}$ and $v \not b$. We claim that

$$
\mathcal{E}_{\text {top }}^{1, p}(v)>0
$$

(13) Here, we use the embedding $W^{1, \ell+1}\left(\mathbb{R}^{\ell+1}\right) \subset \mathrm{VMO}\left(\mathbb{R}^{\ell+1}\right)$ 
Indeed, argue by contradiction and assume that there exists a sequence of maps $\left(v_{j}\right)_{j \in \mathbb{N}}$ in $W_{b}^{1, \ell+1}\left(\mathbb{B}^{\ell}, \mathcal{N}\right)$ such that $v_{j} \sim v, \forall j$ and $\mathcal{E}^{1, p}\left(v_{j}\right) \rightarrow 0$. By the Morrey embedding $W^{1, \ell+1}\left(\mathbb{B}^{\ell}\right) \subset C^{0,1-\ell /(\ell+1)}\left(\overline{\mathbb{B}}^{\ell}\right)$ and the fact that $v_{j}=$ $b$ on $\mathbb{S}^{\ell-1}$, we find that $v_{j} \rightarrow b$ uniformly, and thus, for large $j, v \sim v_{j} \sim b$, a contradiction.

We define for $\rho \in(0,1)$ the map $u_{\rho} \in C^{\infty}\left(\mathbb{R}^{\ell}, \mathcal{N}\right)$ by

$$
u_{\rho}(x) \triangleq \begin{cases}v(x / \rho) & \text { if }|x| \leqslant \rho, \\ b & \text { otherwise }\end{cases}
$$

whose restriction to $\mathbb{B}^{\ell}$, still denoted $u_{\rho}$, satisfies

$$
\begin{aligned}
\mathcal{E}^{1-1 /(\ell+1), \ell+1}\left(u_{\rho}\right) & =\iint_{\mathbb{B}^{\ell} \times \mathbb{B}^{\ell}} \frac{\left|u_{\rho}(y)-u_{\rho}(x)\right|^{\ell+1}}{|y-x|^{2 \ell}} \mathrm{d} y \mathrm{~d} x \\
& \leqslant \iint_{\mathbb{R}^{\ell} \times \mathbb{R}^{\ell}} \frac{\left|u_{\rho}(y)-u_{\rho}(x)\right|^{\ell+1}}{|y-x|^{2 \ell}} \mathrm{d} y \mathrm{~d} x \\
& =\iint_{\mathbb{R}^{\ell} \times \mathbb{R}^{\ell}} \frac{|v(y)-v(x)|^{\ell+1}}{|y-x|^{2 \ell}} \mathrm{d} y \mathrm{~d} x=C_{8} \in(0, \infty) .
\end{aligned}
$$

On the other hand, we clearly have

$$
\lim _{\rho \rightarrow 0+} \mathcal{E}^{1-1 /(\ell+1), \ell+1}\left(u_{\rho}\right)=C_{8} .
$$

Since $u_{\rho} \sim v$, we obtain, from $(2.19)-(2.21)$, that

$$
\mathcal{E}_{\text {ext }}^{1, \ell+1}\left(u_{\rho}\right) \geqslant C_{9} \ln \frac{1}{\rho} \mathcal{E}^{1-1 /(\ell+1), \ell+1}\left(u_{\rho}\right) .
$$

We complete the proof of the theorem in this case via (2.22) and (2.23).

We now deduce Theorem 1.5 from Theorem 1.10.

Proof of Theorem 1.5. - By Theorem 1.10 and an extension argument for fractional Sobolev spaces, there exists a sequence of mappings $\left(u_{j}\right)_{j \in \mathbb{N}}$ in $C^{\infty}\left(\mathbb{R}^{m-1}, \mathcal{N}\right)$ such that for every $j \in \mathbb{N}, u_{j}=b$ on $\mathbb{R}^{m-1} \backslash \mathbb{B}^{m-1}$,

$$
\mathcal{E}^{1-1 / p, p}\left(u_{j}\right) \geqslant C_{10} \text { and } \mathcal{E}_{\text {ext }}^{1, p}\left(u_{j}\left\lceil_{\mathbb{B}^{m-1}}\right) \geqslant 2^{j} \mathcal{E}^{1-1 / p, p}\left(u_{j}\right)\right.
$$

for some constant $C_{10}>0$. We fix the radii $r_{j}>0$ by the condition

$$
r_{j}^{m-p} \mathcal{E}_{\text {ext }}^{1, p}\left(u_{j}\right)=1 .
$$

Since, by assumption $p<m$, we have $r_{j} \leqslant C_{11} 2^{-j /(m-p)}$, so that, in particular,

$$
\sum_{j \in \mathbb{N}} r_{j}^{m-1}<\infty .
$$

Therefore, we may find some $0<\lambda<\infty$ and a sequence of points $\left(a_{j}\right)_{j \in \mathbb{N}}$ in $\mathbb{B}^{m-1}$ converging to a point $a_{*} \in \mathbb{S}^{m-2}$ such that the balls $B\left(a_{j}, \lambda r_{j}\right)$ 
are mutually disjoint and contained in $\mathbb{B}^{m-1}$. We then define the map $u \in$ $C^{\infty}\left(\mathbb{B}^{m-1}, \mathcal{N}\right)$ by setting, for $x \in \mathbb{B}^{m-1}$,

$$
u(x) \triangleq \begin{cases}u_{j}\left(1 /\left(\lambda r_{j}\right)\left(x-a_{j}\right)\right) & \text { if } x \in B\left(a_{j}, \lambda r_{j}\right) \text { for some } j \\ b & \text { otherwise. }\end{cases}
$$

By the superadditivity of the extension energy and (2.25), we have

$$
\mathcal{E}_{\text {ext }}^{1, p}(u) \geqslant \sum_{j \in \mathbb{N}}\left(\lambda r_{j}\right)^{m-p} \mathcal{E}_{\text {ext }}^{1, p}\left(u_{j}\right)=\sum_{j \in \mathbb{N}} \lambda^{m-p}=\infty .
$$

On the other hand, we have, by the almost subadditivity property for Sobolev mappings having disjoint supports [25, Lemma 2.3], (2.24) and (2.25):

$$
\begin{aligned}
\mathcal{E}^{1-1 / p, p}(u) & \leqslant C_{12} \sum_{j \in \mathbb{N}}\left(\lambda r_{j}\right)^{m-p+1} \mathcal{E}^{1-1 / p, p}\left(u_{j}\right) \\
& \leqslant C_{12} \lambda^{m-p+1} \sum_{j \in \mathbb{N}} 2^{-j} r_{j} \\
& \leqslant C_{12} \lambda^{m-p+1} \sum_{j \in \mathbb{N}} 2^{-j}<\infty
\end{aligned}
$$

By $(2.26)$ and $(2.27), u$ is a $W^{1-1 / p, p}\left(\mathbb{B}^{m-1}, \mathcal{N}\right)$ map with no $W^{1, p}\left(\mathbb{B}^{m-1} \times\right.$ $(0,1), \mathcal{N})$ extension.

\section{Construction of extension}

We explain how Theorem 1.6 and Theorem 1.9 follow from existing results on extension for simply-connected manifolds through a lifting argument; this important observation is due to F. Bethuel [4].

Proof of the new cases in Theorem 1.6 and Theorem 1.9. - Let $\pi$ : $\widetilde{\mathcal{N}} \rightarrow \mathcal{N}$ be the universal covering of the manifold $\mathcal{N}$. Since the fundamental group $\pi_{1}(\mathcal{N})$ is finite, the universal covering space $\tilde{\mathcal{N}}$ is compact; in view of the fractional lifting theorem for compact covering spaces [24], for every $u \in W^{1-1 / p, p}\left(\mathbb{B}^{m-1}, \mathcal{N}\right)$ there exists a map $\widetilde{u} \in W^{1-1 / p, p}\left(\mathbb{B}^{m-1}, \widetilde{\mathcal{N}}\right)$ such that $\pi \circ \widetilde{u}=u$ in $\mathbb{B}^{m-1}$ and

$$
\mathcal{E}^{1-1 / p, p}(\widetilde{u}) \leqslant C_{1} \mathcal{E}^{1-1 / p, p}(u),
$$

for some constant $C_{1}$ independent on the mapping $u$.

Since $\widetilde{\mathcal{N}}$ is the universal covering of $\mathcal{N}$, it is simply-connected (that is, $\left.\pi_{1}(\tilde{\mathcal{N}}) \simeq\{0\}\right)$ and it has the same higher-order homotopy groups as $\mathcal{N}$ : for every $j \in\{2, \ldots,\lfloor p-1\rfloor\}$, we have $\pi_{j}(\tilde{\mathcal{N}}) \simeq \pi_{j}(\mathcal{N}) \simeq\{0\}$ (see for 
example [18, Proposition 4.1]). By Theorem 1.4 and Theorem 1.9(1) (applied to the old case where $\left.\pi_{1}(\mathcal{N}) \simeq\{0\}\right)$, there exists a mapping $\widetilde{U} \in$ $W^{1, p}\left(\mathbb{B}^{m-1} \times(0,1), \widetilde{\mathcal{N}}\right)$ with trace $\widetilde{u}$ and such that

$$
\mathcal{E}^{1, p}(\widetilde{U}) \leqslant C_{2} \mathcal{E}^{1-1 / p, p}(\widetilde{u}) .
$$

We conclude by defining $U \triangleq \pi \circ \widetilde{U}$. Since the covering map $\pi$ is a local isometry, we have $u=\pi \circ \widetilde{u}$ on $\mathbb{B}^{m-1}$ and $u=\operatorname{tr}_{\mathbb{B}^{m-1} \times\{0\}} U, U \in$ $W^{1, p}\left(\mathbb{B}^{m-1} \times(0,1), \mathcal{N}\right)$ and

$$
\mathcal{E}^{1, p}(U)=\mathcal{E}^{1, p}(\widetilde{U}) \leqslant C_{2} \mathcal{E}^{1-1 / p, p}(\widetilde{u}) \leqslant C_{2} C_{1} \mathcal{E}^{1-1 / p, p}(u) .
$$

\section{Manifolds on which the problem is open}

The next proposition shows the existence of compact manifolds with finitely many prescribed homotopy groups. This is a straightforward and probably well-known variant of the product of Eilenberg-McShane spaces giving CW complexes with an arbitrary sequence of homotopy groups [18, $\S 4.2]$. The interest of the next proposition is that the resulting space is a compact finite-dimensional manifold.

Proposition 4.1. - If $\ell \in \mathbb{N}$ and $G_{1}, \ldots, G_{\ell}$ are finitely generated groups, and if $G_{2}, \ldots, G_{\ell}$ are abelian, then there exists a $2(\ell+1)$-dimensional compact manifold $\mathcal{N}$ such that for every $j \in\{1, \ldots, \ell\}, \pi_{j}(\mathcal{N})=G_{j}$.

Proof. - We define

$$
X \triangleq K\left(G_{1}, 1\right) \times \cdots \times K\left(G_{\ell}, \ell\right),
$$

where the Eilenberg-McLane space $K\left(G_{j}, j\right)$ is a CW-complex of finite type whose only non-trivial homotopy group is $\pi_{j}\left(K\left(G_{j}, j\right)\right)=G_{j}[18, \S 4.2]$. We then have $\pi_{j}(X)=G_{j}$ for every $j \in\{1, \ldots, \ell\}$. Let $X_{\ell+1}$ be the component of $X$ consisting of cells of dimensions at most $\ell+1$. It follows then that $\pi_{j}\left(X_{\ell+1}\right)=G_{j}$ for every $j \in\{1, \ldots, \ell\}$. Since $X$ is of finite type, $X_{\ell+1}$ is a finite $\mathrm{CW}$-complex, that can be realized as a simplicial complex $K$ of dimension $\ell+1$. We embed $K$ in the Euclidean space $\mathbb{R}^{\nu}$ with $\nu=2 \ell+3$ and we let $\mathcal{N} \triangleq \partial \mathcal{U}$, where $\mathcal{U}$ is a smooth neighborhood of $K$ that retracts on $K$ and such that $\mathcal{U} \backslash K$ retracts on $\mathcal{N}$. Since $K$ is of dimension $\ell+1$, it follows that for every $j \in\{1, \ldots, \ell\}$, any continuous map $f: \mathbb{B}^{j+1} \rightarrow \mathcal{U}$ such that $f\left\lceil_{\mathbb{S}^{j}}\right.$ takes its values in $\mathcal{N}$ is homotopic to a map with values in $\mathcal{U} \backslash K$, and thus $\pi_{j}(\mathcal{N})=\pi_{j}(K)=\pi_{j}\left(X_{\ell+1}\right)=G_{j}$. 


\section{Bibliography}

[1] R. A. Adams \& J. J. F. Fournier, Sobolev spaces, 2 ed., Pure and Applied Mathematics, vol. 140, Academic Press Inc., 2003, xiv +305 pages.

[2] F. Bethuel, "A characterization of maps in $H^{1}\left(\mathbb{B}^{3}, \mathbb{S}^{2}\right)$ which can be approximated by smooth maps", Ann. Inst. Henri Poincaré, Anal. Non Linéaire 7 (1990), no. 4, p. 269-286.

[3] - "The approximation problem for Sobolev maps between two manifolds", Acta Math. 167 (1991), no. 3-4, p. 153-206.

[4] - "A new obstruction to the extension problem for Sobolev maps between manifolds", J. Fixed Point Theory Appl. 15 (2014), no. 1, p. 155-183.

[5] F. Bethuel \& D. Chiron, "Perspectives in nonlinear partial differential equations", in Perspectives in nonlinear partial differential equations, Contemporary Mathematics, vol. 446, American Mathematical Society, 2007, p. 125-152.

[6] F. Bethuel, J.-M. Coron, F. Demengel \& F. Hélein, "A cohomological criterion for density of smooth maps in Sobolev spaces between two manifolds", in Nematics. Mathematical and physical aspects, NATO ASI Series. Series C. Mathematical and Physical Sciences, vol. 332, Kluwer Academic Publishers, 1991, p. 15-23.

[7] F. Bethuel \& F. Demengel, "Extensions for Sobolev mappings between manifolds", Calc. Var. Partial Differ. Equ. 3 (1995), no. 4, p. 475-491.

[8] J. Bourgain, H. Brezis \& P. Mironescu, "Lifting in Sobolev spaces", J. Anal. Math. 80 (2000), p. 37-86.

[9] - " $H^{1 / 2}$ maps with values into the circle: minimal connections, lifting, and the Ginzburg-Landau equation", Publ. Math., Inst. Hautes Étud. Sci. (2004), no. 99, p. $1-115$.

[10] P. Bousquet, A. C. Ponce \& J. Van Schaftingen, "Strong approximation of fractional Sobolev maps", J. Fixed Point Theory Appl. 15 (2014), no. 1, p. 133-153.

[11] H. Brezis \& P. Mironescu, "Sobolev maps to the circle", in preparation.

[12] - "Gagliardo-Nirenberg, composition and products in fractional Sobolev spaces", J. Evol. Equ. 1 (2001), no. 4, p. 387-404.

[13] - "Density in $W^{s, p}(\Omega ; \mathcal{N}) "$, J. Funct. Anal. 269 (2015), no. 7, p. 2045-2109.

[14] H. Brezis \& L. Nirenberg, "Degree theory and BMO", Sel. Math., New Ser. 1 (1995), no. 2, p. 197-263.

[15] E. DiBenedetto, Real analysis, 2 ed., Birkhäuser Advanced Texts. Basler Lehrbücher, Birkhäuser; Springer, 2016, xxxii+596 pages.

[16] E. Gagliardo, "Caratterizzazioni delle tracce sulla frontiera relative ad alcune classi di funzioni in $n$ variabili", Rend. Semin. Mat. Univ. Padova 27 (1957), p. 284-305.

[17] R. HARDT \& F.-H. Lin, "Mappings minimizing the $L^{p}$ norm of the gradient", Commun. Pure Appl. Math. 40 (1987), no. 5, p. 555-588.

[18] A. Hatcher, Algebraic topology, Cambridge University Press, 2002, xii+544 pages.

[19] T. Isobe, "Obstructions to the extension problem of Sobolev mappings", Topol. Methods Nonlinear Anal. 21 (2003), no. 2, p. 345-368.

[20] J.-L. Lions \& J. Peetre, "Sur une classe d'espaces d'interpolation", Publ. Math., Inst. Hautes Étud. Sci. (1964), no. 19, p. 5-68.

[21] V. MAZ'YA, Sobolev spaces with applications to elliptic partial differential equations, 2 ed., Grundlehren der Mathematischen Wissenschaften, vol. 342, Springer, 2011, xxviii+866 pages.

[22] V. MaZ'ya \& T. Shaposhnikova, "On the Brezis and Mironescu conjecture concerning a Gagliardo-Nirenberg inequality for fractional Sobolev norms", J. Math. Pures Appl. 81 (2002), no. 9, p. 877-884. 
[23] P. Mironescu \& E. Russ, "Traces of weighted Sobolev spaces. Old and new", Nonlinear Anal., Theory Methods Appl. 119 (2015), p. 354-381.

[24] P. Mironescu \& J. VAn Schaftingen, "Lifting of fractional Sobolev maps to compact covering spaces", https://arxiv.org/abs/1907.01373, to appear in Anal. PDE.

[25] A. Monteil \& J. Van Schaftingen, "Uniform boundedness principles for Sobolev maps into manifolds", Ann. Inst. Henri Poincaré, Anal. Non Linéaire 36 (2019), no. 2, p. 417-449.

[26] J. NAsh, "The imbedding problem for Riemannian manifolds", Ann. Math. 63 (1956), p. 20-63.

[27] J. Peetre, "A counter-example connected with Gagliardo's trace theorem", Commentat. Math. 2 (1979), p. 277-282.

[28] T. Rivière, "Dense subsets of $H^{1 / 2}\left(\mathbb{S}^{2}, \mathbb{S}^{1}\right)$ ", Ann. Global Anal. Geom. 18 (2000), no. 5 , p. $517-528$.

[29] T. Runst, "Mapping properties of nonlinear operators in spaces of Triebel-Lizorkin and Besov type", Anal. Math. 12 (1986), no. 4, p. 313-346.

[30] R. Schoen \& K. Uhlenbeck, "A regularity theory for harmonic maps", J. Differ. Geom. 17 (1982), no. 2, p. 307-335.

[31] - "Boundary regularity and the Dirichlet problem for harmonic maps", $J$. Differ. Geom. 18 (1983), no. 2, p. 253-268.

[32] S. V. UspenskII , "Imbedding theorems for classes with weights", Tr. Mat. Inst. Steklova 60 (1961), p. 282-303, translation in Trans. Am. Math. Soc. 87 (1970), p. 121-145.

[33] B. White, "Homotopy classes in Sobolev spaces and the existence of energy minimizing maps", Acta Math. 160 (1988), no. 1-2, p. 1-17. 